PROCEEDINGS OF THE

AMERICAN MATHEMATICAL SOCIETY

Volume 138, Number 6, June 2010, Pages 2037-2042

S 0002-9939(09)10223-X

Article electronically published on December 8, 2009

\title{
FIBRATIONS AND STEIN NEIGHBORHOODS
}

\author{
FRANC FORSTNERIC AND ERLEND FORNÆSS WOLD \\ (Communicated by Mei-Chi Shaw)
}

\begin{abstract}
Let $Z$ be a complex space and let $S$ be a compact set in $\mathbb{C}^{n} \times Z$ which is fibered over $\mathbb{R}^{n}$. We give a necessary and sufficient condition for $S$ to be a Stein compactum.
\end{abstract}

\section{Fibrations over totally REAL SETS}

We denote by $\mathcal{O}(Z)$ the algebra of all holomorphic functions on a (reduced, paracompact) complex space $Z$, endowed with the compact-open topology. A compact subset $K$ of $Z$ is said to be a Stein compactum if $K$ has a basis of Stein open neighborhoods. $K$ is $\mathcal{O}(Z)$-convex if

$$
K=\widehat{K}_{\mathcal{O}(Z)}=\left\{z \in Z:|f(z)| \leq \sup _{K}|f|, \forall f \in \mathcal{O}(Z)\right\} .
$$

For the theory of Stein spaces we refer to [10, 11].

Let $Z$ be a complex space, and consider the product $\mathbb{C}^{n} \times Z$ with the projection $\pi: \mathbb{C}^{n} \times Z \rightarrow \mathbb{C}^{n}$. Let $S$ be a compact set in $\mathbb{C}^{n} \times Z$. For every point $\zeta \in \mathbb{C}^{n}$ we let $S_{\zeta}=\{z \in Z:(\zeta, z) \in S\}$ denote the fiber of $S$ over $\zeta$. We are interested in the following:

Question: Under what conditions on the projection $\pi(S) \subset \mathbb{C}^{n}$ and on the fibers $S_{\zeta}$ is $S$ a Stein compactum in $\mathbb{C}^{n} \times Z$ ?

We give the following precise answer under the assumption that the projection $\pi(S)$ is contained in $\mathbb{R}^{n}$, the real subspace of $\mathbb{C}^{n}$.

Theorem 1.1. Let $S$ be a compact set in $\mathbb{C}^{n} \times Z$ whose projection $P=\pi(S)$ is contained in $\mathbb{R}^{n}$. Then $S$ is a Stein compactum in $\mathbb{C}^{n} \times Z$ if and only if for every open neighborhood $U \subset \mathbb{R}^{n} \times Z$ of $S$ there exist open sets $V, \Omega \subset \mathbb{R}^{n} \times Z$, with $S \subset V \Subset \Omega \subset U$, such that for every $u \in P$ the fiber $\Omega_{u}$ is Stein and $\left.\widehat{\left(S_{u}\right)}\right)_{\mathcal{O}\left(\Omega_{u}\right)} \subset V$. The analogous result holds if $\pi(S)$ belongs to a totally real submanifold of $\mathbb{C}^{n}$.

A particular reason for looking at this problem is that a question of this type, for compact sets that are laminated by holomorphic leaves, appears in the recent work by the first author [8]; the relevant result is provided by Corollary 2.2 below.

The following simple example (a thin version of Hartog's figure) shows that it is not enough to assume in Theorem 1.1 that each fiber of $S$ is a Stein compactum.

Received by the editors June 12, 2009, and, in revised form, September 12, 2009.

2010 Mathematics Subject Classification. Primary 32E05, 32E10, 32H02, 32V40.

Key words and phrases. Stein manifold, Stein compactum, holomorphic convexity.

The first author was supported by grants P1-0291 and J1-6173, Republic of Slovenia.

(C)2009 American Mathematical Society 
Example 1.2. Let $Z=\mathbb{C}$ and let $P \subset \mathbb{C}$ denote the real unit interval $P=\{u \in$ $\mathbb{R}: 0 \leq u \leq 1\}$. For $0 \leq u \leq \frac{1}{2}$ let $S_{u}=\{z \in \mathbb{C}:|z| \leq 1\}$, and for $\frac{1}{2} \leq u \leq 1$ let $S_{u}=\left\{z \in \mathbb{C}: \frac{1}{2} \leq|z| \leq 1\right\}$. Clearly each fiber $S_{u}$ is a Stein compactum, but due to the continuity principle $S$ doesn't have a Stein neighborhood basis in $\mathbb{C}^{2}$.

Remark 1.3. In the situation of Theorem 1.1, if $Z$ is a Stein space and if every fiber $S_{u}$ for $u \in \pi(S) \subset \mathbb{R}^{n}$ is $\mathcal{O}(Z)$-convex, then it is easily seen that $S$ is $\mathcal{O}\left(\mathbb{C}^{n} \times Z\right)$ convex, and hence a Stein compactum. Following the proof of Proposition 4.3 in [12] one obtains the following:

Proposition 1.4. Let $\varphi: Z \rightarrow X$ be a holomorphic map from a Stein space $Z$ to a complex manifold $X$. Let $S \subset Z$ be compact and let $Y=\varphi(S) \subset X$. If $y \in Y$ is a peak point for the algebra $\mathcal{O}_{X}(Y)$, then

$$
\widehat{S}_{\mathcal{O}(Z)} \cap S_{y}={\widehat{\left(S_{y}\right)_{\mathcal{O}(Z)}}}
$$

Proof of Theorem 1.1. We shall give the details only when $\pi(S) \subset \mathbb{R}^{n}$; the general case when the projection is contained in a totally real submanifold of $\mathbb{C}^{n}$ is quite similar.

It is easy to see that the conditions are necessary. Indeed, if $S$ is a Stein compactum, then for every open neighborhood $U$ of $S$ in $\mathbb{R}^{n} \times Z$ there exists a Stein neighborhood $\Omega$ of $S$ in $\mathbb{C}^{n} \times Z$ such that $\Omega \cap\left(\mathbb{R}^{n} \times Z\right) \subset U$. Taking $V \Subset \Omega$ to be an open neighborhood of $\widehat{S}_{\mathcal{O}(\Omega)}$ in $\mathbb{R}^{n} \times Z$ we see that the hypotheses of the theorem are satisfied.

Assume now that the conditions hold. For $\epsilon>0$ set

$$
V(\epsilon)=\left\{(u+i v, z) \in \mathbb{C}^{n} \times Z:(u, z) \in V,\|v\|<\epsilon\right\} .
$$

To prove the theorem we shall construct a plurisubharmonic polyhedral neighborhood of $S$ contained in $V\left(\epsilon_{0}\right)$ for a given $\epsilon_{0}>0$.

Choose an open set $V^{\prime}$ in $\mathbb{R}^{n} \times Z$ such that $V \Subset V^{\prime} \Subset \Omega$. We shall need two lemmas.

Lemma 1.5. There exists a positive strongly plurisubharmonic function in an open neighborhood $\widetilde{U}$ of $S$ in $\mathbb{C}^{n} \times Z$.

Proof. Fix a point $s_{0}=\left(u_{0}, z_{0}\right) \in S$. By compactness it suffices to show that there exists a plurisubharmonic function in a neighborhood of $S$ which is strongly plurisubharmonic near $s_{0}$.

Since $V_{u_{0}}^{\prime} \Subset \Omega_{u_{0}}$ and the set $\Omega$ is open in $\mathbb{R}^{n} \times Z$, there exists a $\delta>0$ such that $V_{u}^{\prime} \Subset \Omega_{u_{0}}$ for all $u$ in the ball $B\left(u_{0}, 2 \delta\right)=\left\{\left\|u-u_{0}\right\|<2 \delta\right\}$.

As $W=\left(B(u, 2 \delta)+i \mathbb{R}^{n}\right) \times \Omega_{u_{0}}$ is Stein, there exists a positive strongly plurisubharmonic function $\rho_{1}$ on $W$. We may assume that $\rho_{1}\left(s_{0}\right)=1$.

Choose $M \in \mathbb{R}$ such that for any $u \in \partial B\left(u_{0}, \delta\right)$ and any $z \in V_{u}^{\prime}$ we have that $\rho_{1}(u, z)<M$. Let $\rho_{2}$ be a plurisubharmonic function on $\mathbb{C}^{n}$ such that $\rho_{2}\left(u_{0}\right)=0$ and $\rho_{2}(u)>M$ for all $u \in \partial B\left(u_{0}, \delta\right)$. If $\epsilon>0$ is chosen small enough, then the function $\rho=\max \left\{\rho_{1}, \rho_{2}\right\}$ is well defined and plurisubharmonic on $V^{\prime}(\epsilon)$ and is strongly plurisubharmonic near the point $s_{0}$.

By shrinking the set $U \supset S$ we may assume that $U=\widetilde{U} \cap\left(\mathbb{R}^{n} \times Z\right)$, where $\widetilde{U}$ satisfies the conclusion of Lemma 1.5. 
Lemma 1.6. For any point $q$ belonging to the boundary of $V$ in $\mathbb{R}^{n} \times Z$ there exists an $\epsilon>0$ and a continuous plurisubharmonic function $\rho$ on $V^{\prime}(\epsilon)$ such that $\rho(q)=2$ and $\rho<\frac{1}{2}$ on $S$.

Proof. If $\pi(q) \notin \pi(S)=P$, we may find a holomorphic function $g \in \mathcal{O}\left(\mathbb{C}^{n}\right)$ such that $\rho=|g \circ \pi|$ will work.

Assume now that $q=(u, z)$ with $u \in P$. Since ${\widehat{\left(S_{u}\right)}}_{\mathcal{O}\left(\Omega_{u}\right)} \subset V_{u}$ by the assumption, there is a holomorphic function $f \in \mathcal{O}\left(\Omega_{u}\right)$ such that $f(z)=2$ and $|f|<\frac{1}{2}$ on $S_{u}$. By continuity there is a $\delta>0$ such that for all $u^{\prime} \in \overline{B(u, \delta)}$ we have that $V_{u^{\prime}}^{\prime} \Subset \Omega_{u}$ and $|f|<\frac{1}{2}$ on $S_{u^{\prime}}$. Let $M \in \mathbb{R}$ be such that $|f| \leq M$ on $V_{u^{\prime}}^{\prime}$ for all $u^{\prime} \in \overline{B(u, \delta)}$. We consider $f$ as a holomorphic function on $\mathbb{C}^{n} \times \Omega_{u}$ which is independent of the first variable.

Let $\chi \in \mathcal{C}_{0}^{\infty}(B(u, \delta))$ be a smooth function with compact support such that $0 \leq \chi \leq 1$ and $\chi(u)=1$. Let $g \in \mathcal{O}\left(\mathbb{C}^{n}\right)$ be a holomorphic function approximating $\chi$ close enough on $\overline{B(u, \delta)}$ such that $\|g\|_{\partial B(u, \delta)}<\frac{1}{3 M}$ and $g(u)=1$. Then $\rho_{1}:=|f \cdot g|$ is plurisubharmonic on $\left[\overline{B(u, \delta)} \oplus \mathrm{i} \mathbb{R}^{\mathrm{n}}\right] \times \Omega_{u}, \rho_{1}(q)=|f(z)|=2$, and $\rho_{1}(w)<\frac{1}{3}$ for all $w=\left(u^{\prime}, z\right)$ such that $u^{\prime} \in \partial B(u, \delta)$ and $z \in V_{u^{\prime}}^{\prime}$.

Let $h \in \mathcal{O}\left(\mathbb{C}^{n}\right)$ be a holomorphic function such that $h(u)=0,0 \leq|h| \leq \frac{1}{2}$ on $B(u, \delta)$, and $\frac{1}{3}<|h|<\frac{1}{2}$ on $\mathbb{R}^{n} \backslash B(u, \delta)$. Such $h$ exists since continuous functions on $\mathbb{R}^{n}$ can be approximated uniformly by entire functions on $\mathbb{C}^{n}$ (see for instance [14).

The function $\rho_{2}:=|h \circ \pi|$ is then plurisubharmonic on $\mathbb{C}^{n} \times Z$.

If $\epsilon>0$ is chosen small enough, then the function $\rho=\max \left\{\rho_{1}, \rho_{2}\right\}$ is well defined on $V^{\prime}(\epsilon)$ and it satisfies the conclusion of Lemma 1.6.

We can now complete the proof of Theorem 1.1. By compactness and Lemma 1.6 there exist an $\epsilon>0$, with $2 \epsilon<\epsilon_{0}$, and finitely many plurisubharmonic functions $\rho_{1}, \ldots, \rho_{m}$ on $V^{\prime}(2 \epsilon)$ such that $\rho_{j}<\frac{1}{2}$ on $S$ for $j=1, \ldots, m$ and such that for every $q=(u, z) \in \partial V \subset \mathbb{R}^{n} \times Z$ we have $\rho_{j}(q)>\frac{3}{2}$ for at least one $j \in\{1, \ldots, m\}$.

Denote the variable on $\mathbb{C}^{n}$ by $\zeta=u+i v$. If we replace $\rho_{j}$ by $\rho_{j}+C|v|^{2}$ for a sufficiently large $C>0$, then we have that

$$
\partial[V(\epsilon)] \subset \bigcup_{j=1}^{m}\left\{w \in V^{\prime}(2 \epsilon): \rho_{j}(w)>\frac{3}{2}\right\} .
$$

Let $W$ denote the set

$$
W=\left\{w \in V^{\prime}(2 \epsilon): \rho_{j}(w)<1, j=1, \ldots, m\right\} .
$$

Let $W_{0}$ be the union of all connected components of $W$ which intersect $S$. Then $S \subset W_{0}$ and $W_{0}$ is relatively compact in $V(\epsilon)$.

Let $\varphi \in \mathcal{C}^{\infty}((-\infty, 1))$ be a convex increasing function such that $\varphi(t) \rightarrow+\infty$ as $t \rightarrow 1$. Let $\widetilde{\rho}_{j}=\varphi \circ \rho_{j}$ for $j=1, \ldots, m$. Then

$$
\rho:=\max _{1 \leq j \leq m} \widetilde{\rho}_{j}
$$

is a plurisubharmonic exhaustion function of $W_{0}$. By Lemma 1.5 we may add to $\rho$ a strongly plurisubharmonic function and get a strongly plurisubharmonic exhaustion function of $W_{0}$, and hence $W_{0}$ is Stein according to Narasimhan's theorem [13]. Since $\bar{W}_{0} \subset V\left(\epsilon_{0}\right)$, this concludes the proof. 
Corollary 1.7. Assume that $S$ is a compact set in $\mathbb{R}^{n} \times Z$ such that for any open neighborhood $U \subset \mathbb{R}^{n} \times Z$ of $S$ and for each $u \in \pi(S) \subset \mathbb{R}^{n}$ there exist a Stein neighborhood $\Omega_{u} \Subset U_{u}$ of the fiber $S_{u}$ and a number $\delta>0$ such that $S_{u^{\prime}}$ is holomorphically convex in $\Omega_{u}$ for each $u^{\prime}$ with $\left\|u-u^{\prime}\right\| \leq \delta$. Then $S$ is a Stein compactum in $\mathbb{C}^{n} \times Z$.

Proof. By compactness there is a finite number of $u_{j}$ 's, $\Omega_{u_{j}}$ 's and $\delta_{j}$ 's such that $\left\{B\left(u_{j}, \delta_{j}\right)\right\}$ is an open cover of $\pi(S)$ in $\mathbb{R}^{n}$. If all $\delta_{j}$ 's are small enough we get that $\bigcup_{j} B\left(u_{j}, \delta_{j}\right) \times \Omega_{u_{j}}$ is contained in $U$.

Define a neighborhood $\Omega$ of $S$ in $\mathbb{R}^{n} \times Z$ as follows. For a point $u \in \overline{B\left(u_{j}, \delta_{j}\right)}$ let the fiber $\Omega_{u}$ consist of the intersection of all $\Omega_{u_{k}}$ such that $u$ is also contained in $\overline{B\left(u_{k}, \delta_{k}\right)}$. Then $\Omega$ is an open neighborhood of $S$ contained in $U$, and $S_{u}$ is holomorphically convex in $\Omega_{u}$ for every $u$. Let $V \Subset \Omega$ be any open neighborhood that contains $S$. The conditions in Theorem 1.1 are satisfied and hence the corollary follows.

\section{Stein neighborhoods of Certain laminated sets}

According to Siu's theorem [15] every closed Stein subvariety of a complex space admits an open Stein neighborhood in that space. For simpler proofs and generalizations to $q$-convex subspaces see 2, 3.

The following generalization of Siu's theorem is proved in [6, Theorem 2.1] (for the last stetement see also [7, Theorem 1.2]).

Theorem 2.1. Let $X$ be a closed Stein subvariety of complex space $Z$. Assume that $S$ is a compact set in $Z$ that is $\mathcal{O}(\Omega)$-convex in an open Stein domain $\Omega \subset Z$ containing $S$ and such that $S \cap X$ is $\mathcal{O}(X)$-convex. Then for every open set $U$ in $Z$ containing $S \cup X$ there exists an open Stein domain $V$ in $Z$ such that $S \cup X \subset V \subset U$ and $S$ is $\mathcal{O}(V)$-convex.

In the remainder of the article we consider the following situation: $Z$ is a complex space, $X$ is a Stein space, $h: Z \rightarrow X$ is a holomorphic submersion onto $X, K$ is a compact $\mathcal{O}(X)$-convex subset of $X$, and $P$ is a compact set in $\mathbb{R}^{n}$ (considered as the real subspace of $\left.\mathbb{C}^{n}\right)$.

The following corollary is used in an essential way in the proof of the main result in 8 . to the effect that all Oka properties of a complex manifold are equivalent to each other.

Corollary 2.2 (Assumptions as above). Let $f: P \times X \rightarrow Z$ be a continuous map such that $h \circ f(p, x)=x$ for all $(p, x) \in P \times X$ and such that $f_{p}=f(p, \cdot): X \rightarrow Z$ is holomorphic for every $p \in P$. Then the set

$$
\Sigma=\{(p, f(p, x)): p \in P, x \in K\}
$$

admits an open Stein neighborhood $\Theta$ in $\mathbb{C}^{n} \times Z$ such that $\Sigma$ is $\mathcal{O}(\Theta)$-convex.

Proof. For every $p \in P$ the set $V_{p}=f_{p}(X)$ is a closed Stein subvariety of $Z$, and $\Sigma_{p}=f_{p}(K) \subset V_{p}$ (the fiber of $\Sigma$ over $p$ ) is $\mathcal{O}\left(V_{p}\right)$-convex.

Fix $p \in P$. By Siu's theorem [15] there exists an open Stein neighborhood $\Omega \subset Z$ of $\Sigma_{p}$. If $q \in P$ is sufficiently near $p$, then, due to the continuity of $f$, we have that $\Sigma_{q} \subset V_{q} \cap \Omega$. Since $\Sigma_{q}$ is $\mathcal{O}\left(V_{q}\right)$-convex, it is also $\mathcal{O}\left(V_{q} \cap \Omega\right)$-convex. As $V_{q} \cap \Omega$ is a closed subvariety of the Stein domain $\Omega$, it follows that $\Sigma_{q}$ is also $\mathcal{O}(\Omega)$-convex. (Indeed, for any point $z \in \Omega \backslash V_{q}$ there exists by Cartan's theorem a holomorphic 
function on $\Omega$ that equals one at $z$ and that vanishes on $V_{q} \cap \Omega$; hence no such point can belong to the $\mathcal{O}(\Omega)$-hull of $\Sigma_{q}$.)

This shows that $\Sigma$ satisfies the assumptions of Corollary 1.7 and hence it admits a basis of open Stein neighborhoods in $\mathbb{C}^{n} \times Z$.

Since $P$ is contained in $\mathbb{R}^{n}$, we have that $\left.\mathcal{O}\left(\mathbb{C}^{n}\right)\right|_{P}$ is dense in $\mathcal{C}(P)$, the space of complex valued continuous functions on $P$ (see [14]). By using cutoff functions in the Euclidean variable and approximating them sufficiently well by entire functions we see that $\Sigma$ is $\mathcal{O}(\Theta)$-convex in every open Stein set $\Theta \subset \mathbb{C}^{n} \times Z$ containing $\Sigma$. This completes the proof.

Remark 2.3. The set $\Sigma$ in Corollary 2.2 is laminated by the graphs of the holomorphic functions $f_{p}$ over $K$, depending continuously on the parameter $p \in P$. This lamination is rather simple since it admits a global product structure. In general, the existence of Stein neighborhoods of sets that are foliated (or laminated) by complex submanifolds is a rather subtle issue as is seen in the case of worm type Levi-flat hypersurfaces (see [1, 4, 9]). Corollary 2.2 extends to the more general situation when the leaves $\Sigma_{p}$ are not necessarily graphs, but compact holomorphically convex subsets in a continuously moving family of Stein subvarieties of $Z$.

In the final result we shall need the following well known implication of Rossi's local maximum modulus principle (see e.g. [5. Lemma 6.5] for the case $\Omega=\mathbb{C}^{N}$; the general case easily reduces to this one since we can replace $\Omega$ by a relatively compact Stein subset which then embeds as a closed complex subvariety in a Euclidean space).

Lemma 2.4. Assume that $V$ is a closed complex subvariety of a Stein space $\Omega$. If $S$ is a compact $\mathcal{O}(\Omega)$-convex subset of $\Omega$ and if $K$ is a compact $\mathcal{O}(V)$-convex subset of $V$ such that $S \cap V \subset K$, then $K \cup S$ is $\mathcal{O}(\Omega)$-convex.

Our final result furnishes a parametric version of Theorem 2.1.

Corollary 2.5. Assume the situation of Corollary 2.2. Set $V_{p}=f_{p}(X) \subset Z$ for $p \in P$, and let $\Sigma \subset P \times Z$ be the compact set (2.1) with the fibers $\Sigma_{p}=f_{p}(K) \subset V_{p}$. Let $S$ be a compact set in $P \times Z \subset \mathbb{C}^{n} \times Z$, with fibers $S_{p}(p \in P)$, such that

(i) $S_{p} \cap V_{p} \subset \Sigma_{p}$ and $S_{p} \cap V_{p}$ is $\mathcal{O}\left(V_{p}\right)$-convex for each $p \in P$, and

(ii) $S$ is $\mathcal{O}(\Theta)$-convex in an open Stein domain $\Theta \subset \mathbb{C}^{n} \times Z$.

Then $\Sigma \cup S$ is a Stein compact in $\mathbb{C}^{n} \times Z$.

Proof. Fix $p \in P$. By Theorem 2.1 the set $\left(\{p\} \times V_{p}\right) \cup S$ admits an open Stein neighborhood $\Omega \subset \mathbb{C}^{n} \times Z$ such that $S$ is $\mathcal{O}(\Omega)$-convex. It follows that the fiber $S_{q}$ is $\mathcal{O}\left(\Omega_{q}\right)$-convex for every $q \in P$. (Here $\Omega_{q}$ denotes the fiber of $\Omega$ over the point $q \in P$.)

Choose an open Stein neighborhood $\Theta \subset Z$ of the compact set $\Sigma_{p} \cup S_{p}$ such that $\bar{\Theta}$ is compact and contained in $\Omega_{p}$. Since $S$ is compact, $\Omega$ is open and $f$ is continuous, we have for all $q \in P$ sufficiently near $p$ that

$$
\Sigma_{q} \cup S_{q} \subset \Theta \subset \Omega_{q} .
$$

For such $q$ we see as in the proof of Corollary 2.2 that $\Sigma_{q}$ is $\mathcal{O}(\Theta)$-convex. Since $S_{q}$ is also $\mathcal{O}\left(\Omega_{q}\right)$-convex and hence $\mathcal{O}(\Theta)$-convex, Lemma 2.4 now shows that $\Sigma_{q} \cup S_{q}$ is $\mathcal{O}(\Theta)$-convex for all $q \in P$ sufficiently near $p$. The conclusion now follows from Corollary 1.7 


\section{REFERENCES}

1. Bedford, E., Fornæss, J. E., Domains with pseudoconvex neighborhood systems. Invent. Math., 47 (1978), 1-27. MR0499316 (58:17215)

2. Colţoiu, M., Complete locally pluripolar sets. J. Reine Angew. Math., 412 (1990), 108-112. MR 1074376 (91h:32010)

3. Demailly, J.-P., Cohomology of $q$-convex spaces in top degrees. Math. Z., 204 (1990), 283-295. MR.1055992 (91e:32014)

4. Diederich, K., Fornæss, J.-E., Pseudoconvex domains: an example with nontrivial Nebenhülle. Math. Ann., 225 (1977), 275-292. MR0430315 (55:3320)

5. Forstnerič, F., Interpolation by holomorphic automorphisms and embeddings in $\mathbb{C}^{n}$. J. Geom. Anal., 9 (1999), 93-118. MR.1760722 (2001g:32040)

6. Forstnerič, F., Extending holomorphic mappings from subvarieties in Stein manifolds. Ann. Inst. Fourier, 55 (2005), 733-751. MR2149401 (2006c:32012)

7. Forstnerič, F., The Oka principle for sections of stratified fiber bundles. Pure Appl. Math. Quarterly (special issue in honor of Joseph J. Kohn), 6 (2010), no. 3, 843-874.

8. Forstnerič, F., Oka manifolds. C. R. Acad. Sci. Paris, Ser. I, 347 (2009), 1017-1020.

9. Forstnerič, F., Laurent-Thiébaut, C., Stein compacts in Levi-flat hypersurfaces. Trans. Amer. Math. Soc., 360 (2008), no. 1, 307-329. MR2342004 (2008i:32055)

10. Gunning, R. C., Rossi, H., Analytic Functions of Several Complex Variables. Prentice-Hall, Englewood Cliffs, NJ, 1965. MR0180696 (31:4927)

11. Hörmander, L., An Introduction to Complex Analysis in Several Variables. Third ed., NorthHolland, Amsterdam, 1990. MR 1045639 (91a:32001)

12. Manne, P. E., Wold, E. F., Øvrelid, N., Carleman approximation by entire functions on Stein manifolds. Preprint, University of Oslo (2008).

13. Narasimhan, R., The Levi problem for complex spaces. Math. Ann., 142 (1961), 355-365. MR.0148943 (26:6439)

14. Scheinberg, S., Uniform approximation by entire functions. J. Analyse. Math., 29 (1976), 16-18. MR0508100 (58:22664)

15. Siu, J.-T., Every Stein subvariety admits a Stein neighborhood. Invent. Math., 38 (1976), 89-100. MR0435447(55:8407)

Institute of Mathematics, Physics and Mechanics, University of Ljubljana, JadranSKa 19, 1000 Ljubluana, Slovenia

E-mail address: franc.forstneric@fmf.uni-lj.si

Matematisk Institutt, Universitetet i Oslo, Postboks 1053 Blindern, 0316 Oslo, NORWAY

E-mail address: erlendfw@math.uio.no 